\title{
Materiales didácticos demostrativos y el aprendizaje de la matemática en los alumnos universitarios
}

\author{
Demonstrative didactic materials and the learning of mathematics in students in university \\ Marco Niels Gonzales Romero'
}

\begin{abstract}
RESUMEN
Objetivo: Determinar la influencia del uso de materiales didácticos demostrativo en el aprendizaje de la matemática en los estudiantes de la Universidad Nacional Santiago Antúnez de Mayolo Huaraz, 2017. Materiales y Métodos: se aplicó en estudio cuantitativo de tipo casual explicativa. La población constituida por 46 estudiantes del III tercer ciclo de la Facultad de Educación. Se trabajo con dos grupos: Uno de control y otro experimental. Se aplicó encuesta pre y post, a través de un cuestionario de once preguntas. Resultados: El $53 \%$ de los estudiantes indica que el docente siempre emplea materiales didácticos en las secciones de clases, el $34 \%$ aprenden formulas matemática usando materiales didácticos, el $43 \%$ indican que la mejor forma de aprender una propiedad matemática es con el material didáctico, el $74 \%$ opinan que los materiales didácticos deben ser utilizados por los docentes, estudiantes y el $71 \%$ indican que es buena la enseñanza de las propiedades matemáticas en los materiales didácticos demostrativo. Conclusiones: Existe una influencia positiva de los materiales didácticos demostrativos hacia la mejora de los aprendizajes de la matemática.
\end{abstract}

Palabras clave: Didáctica, materiales didácticos, argumentación, razonamiento, demostraciones.

\begin{abstract}
Objective: To determine the influence of the use of demonstrative teaching materials in the learning of mathematics in the students of the National University Santiago Antúnez de Mayolo Huaraz, 2017. Materials and methods: it was applied in a quantitative study of an explanatory casual type. The population constituted by 46 students of the III third cycle of the Faculty of Education. I work with two groups: one of control and another experimental. A pre and post survey was applied through an eleven questionnaire. Results: $53 \%$ of the students indicate that the teacher always uses didactic materials in the class sections, $34 \%$ learn mathematical formulas using didactic materials, $43 \%$ indicate that the best way to learn a mathematical property is with the didactic material , $74 \%$ believe that teaching materials should be used by teachers, students and $71 \%$ indicate that the teaching of mathematical properties in demonstrative teaching materials is good. Conclusions: There is a positive influence of demonstration teaching materials towards the improvement of mathematics learning.
\end{abstract}

Keywords: Didactic, didactic materials, argumentation, reasoning, demonstrations.

\section{INTRODUCCIÓN}

Esta investigación es una propuesta innovadora y está dirigida a todos los docentes y público en general que están preocupados por lograr que sus estudiantes desarrollen habilidades matemáticas dentro de las aulas y fuera de ellas trabajando con materiales didácticos creativos e innovadores.

En este contexto los materiales didácticos optimizan la capacidad de demostración de axiomas, propiedades matemáticas, ya que en un material tangible bien elaborado (Valerio, 2000) ayuda a manipular, observar y a interpretar un postulado matemático. La demostración es el fundamento de la misma matemática, como lo señala Hanna y Jahnke (1996) "la demostración es una característica esencial de la matemática y como tal debería ser un componente clave de la educación matemática". Contribuye Balacheff, (1998); mencionando que las demostraciones desarrollan las operaciones mentales generales como abstraer, concretar, analizar, sintetizar, comparar, clasificar, particularizar, generalizar, interpretar, argumentar, fundamentar, inferir, refutar, deducir, valorar las mismas demostraciones. A ello los niveles de desarrollo del pensamiento de los esposos Van Hiele (1986) deben estar presentes en el aprendizaje de la matemática.

\section{MATERIALES Y MÉTODOS}

El trabajo de investigación es un estudio cuantitativo, de tipo casual explicativa ya que se manipulará la variable independiente (materiales didácticos demostrativos) para obtener los resultados propuestos (aprendizaje de la matemática). La población de estudio estuvo constituida 46 alumnos, conformado por dos grupos de estudiantes del tercer ciclo de la facultad de Educación de la Universidad Nacional "Santiago Antúnez de Mayolo" de Huaraz-Ancash. Se trabajó con dos grupos, uno el control y el otro experimental. La división se hizo en base al criterio de comparación de muestras 'Docente de Matemáticas. Institución Educativa: Gran Unidad Escolar “Mariscal Toríbio de Luzuriaga”. Huaraz, E-mail: mngr_22hotmail.com independientes. Para el recojo de información de las variables independiente (Materiales Didácticos Demostrativos) se utilizó la encuesta en base a un cuestionario, mientras que para la variable dependiente (logro de competencias matemáticas) se usó el pre y pos test en base a los indicadores de aprendizajes.

\section{RESULTADOS}

Los resultados obtenidos en base a las distintas dimensiones a) Materiales concretos: Bloques de tecnopor, b) Elaboración de materiales concretos, c) Importancia de los Niveles de demostración, se reflejan en las siguientes tablas, las cuales fueron organizadas en base a las preguntas del cuestionario.

El primer grupo conformado por las cuatro primeras preguntas, evaluaban el indicador Materiales concretos: Bloques de tecnopor, las cuatro preguntas siguientes, evalúan el indicador Elaboración de Materiales Concretos, y finalmente las 3 últimas preguntas, se ajustan al criterio de Importancia de los niveles de demostración.

Pregunta $\mathrm{N}^{\circ} 1$ : ¿El docente de matemáticas utiliza materiales didácticos en clase?

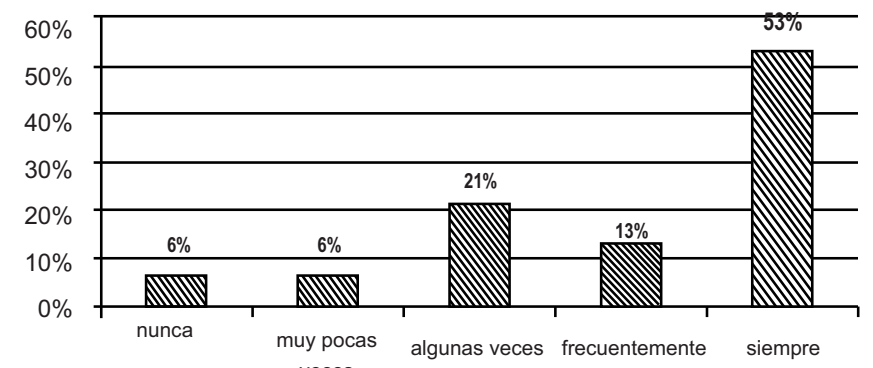

Figura1. El docente y el uso de materiales.

$$
\text { Recibido: 01/11/18 Aprobado: 15/11/18 }
$$


Se aprecia en la Figura1 que el $53 \%$ de los estudiantes encuestados, afirman que siempre el docente universitario de matemáticas emplea materiales didácticos en las sesiones de clase y solo un $6 \%$ afirma que el docente universitario nunca usa materiales didácticos.

Pregunta $\mathbf{N}^{\circ}$ 2: ¿Qué entiendes por demostración matemática?

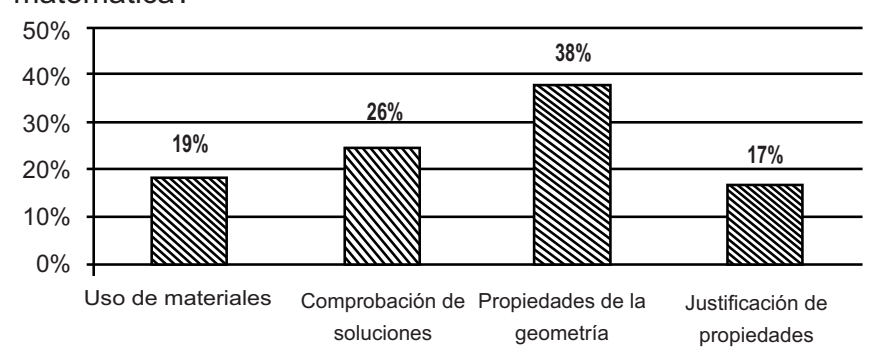

Figura2. El docente y las demostraciones.

Se observa en la Figura 2 que el $83 \%$ de estudiantes encuestados no saben al menos cual es la idea de Demostración matemática y solo un $17 \%$ de los encuestados marco la alternativa "D" que dice que es un procedimiento que justifica las propiedades matemáticas.

Pregunta $\mathbf{N}^{\circ}$ 3: ¿Cómo sabes que las fórmulas que te enseñan son correctas?

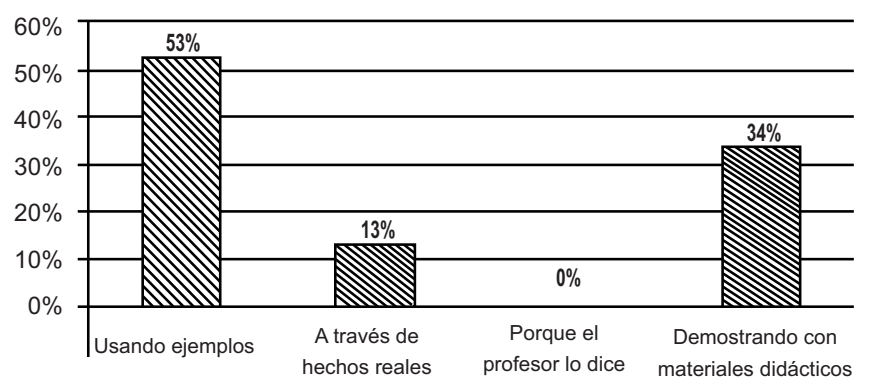

Figura 3.Demostraciones en el aula.

Por medio de la Figura 3, se observa que el $53 \%$ de los estudiantes encuestados afirman que las fórmulas matemáticas se comprenden y aprenden mejor usando ejemplos, un $13 \%$ a través de hechos reales y un $34 \%$ aprenden las fórmulas matemáticas usando materiales didácticos.

Pregunta $\mathrm{N}^{\circ}$ 4: ¿Cómo sabes que las fórmulas que te enseñan son correctas?

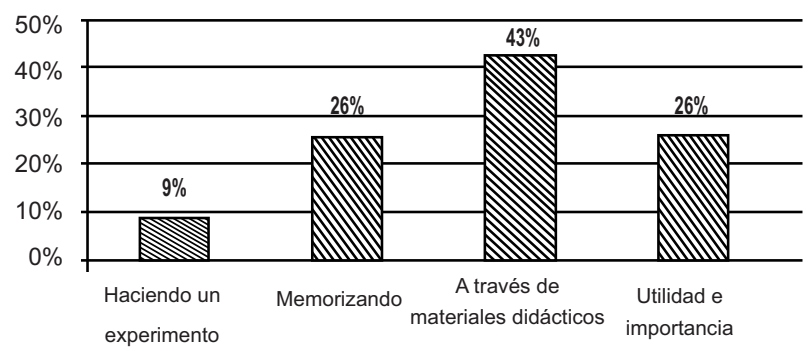

Figura 4. Las fórmulas matemáticas.

En la Figura 4 se observa que el $9 \%$ de los estudiantes encuestados responden que la mejor forma de aprender una propiedad matemática es realizando experimentos, un $26 \%$ usando ejemplos hasta memorizarlos; una mayoría de estudiantes que son el $43 \%$ encuestados lo harían con el material didáctico y un $26 \%$ de estudiantes conociendo su importancia y utilidad de la propiedad.
Pregunta $\mathrm{N}^{\circ}$ 5: ¿Cuándo el docente utiliza materiales didácticos, con qué objetivo las usas?

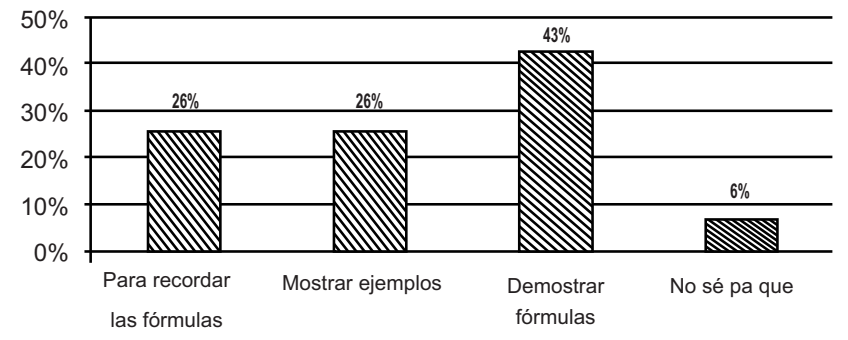

Figura 5.Propósito de las demostraciones.

En la Figura 5 se observa que el $26 \%$ de los estudiantes encuestados responde que el objetivo con que usa materiales didácticos el docente es para que recuerden formulas, un $26 \%$ afirman que lo hace para mostrar ejemplos, una mayoría de estudiantes que es el $43 \%$ lo usa para demostrar fórmulas matemáticas y un $9 \%$ no sabe con qué fin los usa

Pregunta $\mathbf{N}^{\circ} 6$ : ¿Es necesario demostrar las fórmulas en clase?

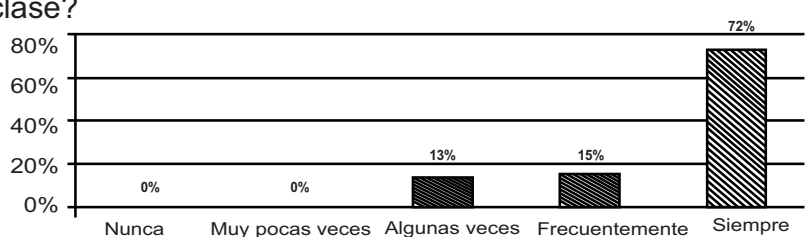

Figura 6. Demostrar fórmulas en clase.

De la Figura 6, las respuestas a la pregunta si es necesario demostrar las formulas en clase, un $13 \%$ respondieron algunas veces, un $15 \%$ opinan que se debe hacer frecuentemente y una mayoría de encuestados que son el $72 \%$ responden que siempre se debe demostrar las fórmulas matemáticas en clase.

Los estudiantes afirman que es importante demostrar las fórmulas que se aprenden en las sesiones de clase.

Pregunta $\mathrm{N}^{\circ}$ 7: $\mathrm{Si}$ el profesor demuestra utilizando materiales didácticos la propiedad de la suma de ángulos internos de un triángulo, ¿Cuál sería tu opinión?

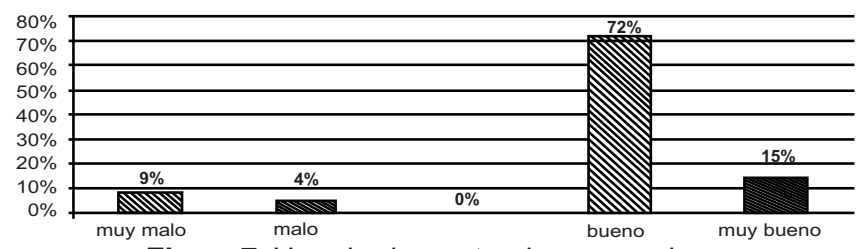

Figura7. Uso de demostraciones en clase.

En la Figura 7 se muestra los resultados a la pregunta, donde $9 \%$ cree que es muy malo, un $4 \%$ malo, a un $72 \%$ responden que sería buena la demostración de propiedades con materiales didácticos y un $15 \%$ dice que sería muy bueno. La mayoría de estudiantes afirma que es bueno que el docente demuestre una propiedad de la matemática en clases.

Pregunta $\mathrm{N}^{\circ}$ 8: ¿Cómo enseña una fórmula matemática tu docente de matemática?

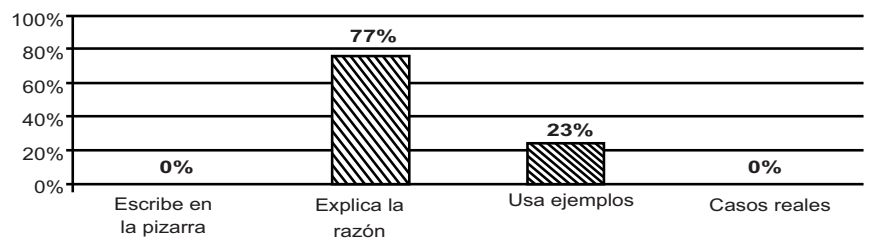

Figura 8: El docente y el uso fórmulas en clase. 
En la Figura 8 se aprecia que el $77 \%$ de los estudiantes encuestados afirmaron que el docente universitario explica siempre el por qué y la razón de una propiedad matemática, y el $23 \%$ de los encuestados respondió que el docente explica las propiedades matemáticas usando diferentes ejemplos aplicativos. La mayoría de los estudiantes afirman que el docente enseña una fórmula matemática a través de ejemplos y señalando su razón.

Pregunta $N^{\circ}$ 9: ¿Cuál es más importante para usted?

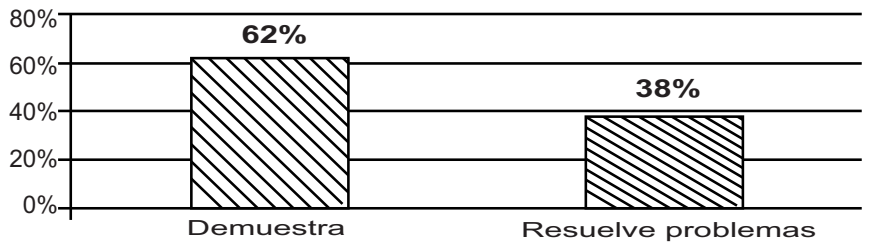

Figura 9: Las demostraciones y su importancia.

En la Figura 9 se muestran que el $62 \%$ de los estudiantes encuestados respondieron que es más importante la demostración de fórmulas matemáticas y un 38\% respondió que es más importante la resolución de problemas. Para la mayoría de estudiantes es más importante la demostración de las fórmulas matemáticas que la resolución de problemas.

Pregunta $\mathrm{N}^{\circ}$ 10: El uso de materiales didácticos demostrativos debe ser manejado por:

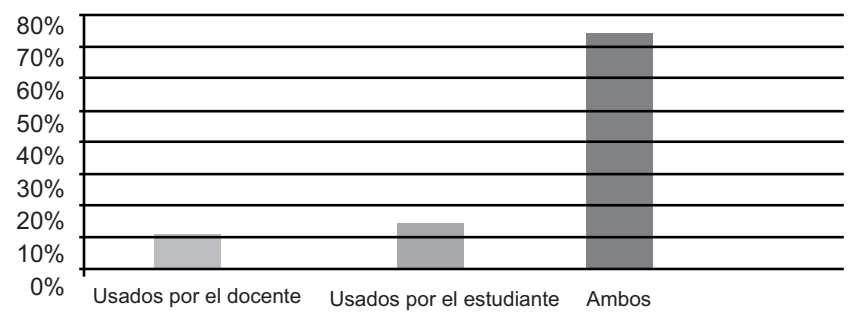

Figura 10: Los materiales didácticos.

Tabla 1. Uso de materiales didácticos demostrativos

Prueba de muestras independientes

\begin{tabular}{|c|c|c|c|c|c|c|c|c|c|c|}
\hline \multicolumn{11}{|c|}{ Prueba de muestras independientes } \\
\hline \multicolumn{6}{|c|}{ Prueba de Levene de igualdad devarianzas } & \multicolumn{5}{|c|}{ prueba t para la igualdad de medias } \\
\hline & & \multirow[t]{2}{*}{$\mathrm{F}$} & \multirow[t]{2}{*}{ Sig. } & \multirow[t]{2}{*}{$\mathrm{t}$} & \multirow[t]{2}{*}{ gl } & \multirow{2}{*}{$\begin{array}{l}\text { Sig. } \\
\text { (bilateral) }\end{array}$} & \multirow{2}{*}{$\begin{array}{c}\text { Diferenc } \\
\text { ia } \\
\text { de } \\
\text { medias }\end{array}$} & \multirow{2}{*}{$\begin{array}{l}\text { Diferencia } \\
\text { de error } \\
\text { estándar }\end{array}$} & \multicolumn{2}{|c|}{$95 \%$ de intervalo de confianza de la diferencia } \\
\hline & & & & & & & & & Inferior & Superior \\
\hline \multirow[b]{2}{*}{ Pretest } & $\begin{array}{c}\text { Se } \\
\text { asumen } \\
\text { varianzas } \\
\text { iguales }\end{array}$ & 9,250 & ,004 & 1,101 & 44 & ,027 & 1,174 & 1,067 &,- 976 & 3,323 \\
\hline & $\begin{array}{c}\text { No se } \\
\text { asumen } \\
\text { varianzas } \\
\text { iguales }\end{array}$ & & & 1,101 & 33,132 & ,019 & 1,174 & 1,067 &,- 996 & 3,343 \\
\hline \multirow{2}{*}{ Postest } & $\begin{array}{c}\mathrm{Se} \\
\text { asumen } \\
\text { varianzas } \\
\text { iguales }\end{array}$ & 3,051 & ,088 & 6,091 & 44 &, 030 & $-4,391$ & ,721 & $-5,844$ & $-2,938$ \\
\hline & $\begin{array}{c}\text { No se } \\
\text { asumen } \\
\text { varianzas } \\
\text { iguales }\end{array}$ & & & 6,091 & 38,266 & ,007 & $-4,391$ & $-5,851$ & $-5,851$ & $-2,932$ \\
\hline
\end{tabular}

Para $\infty=0,05$ se obtuvo el $p$ - valor de 0.27 y 0.19 para el pretest y 0.30, 0.07 para el postest adoptando la decisión de aceptar la hipótesis alterna, por lo cual se puede afirmar que: si se utiliza adecuadamente los materiales didácticos demostrativos en las sesiones de clase mejorará el
En la figura 10 se observa que el $11 \%$ de los estudiantes encuestados afirma que los materiales didácticos solo debe usarlo el docente, un $15 \%$ que solo deben usarlo los estudiantes y la mayoría de estudiantes que son el $74 \%$ afirma que debe ser usado por docentes y estudiantes. Los estudiantes en su mayoría afirman que los materiales didácticos deben ser manipulados no solo por el docente, sino también por los propios estudiantes.

Pregunta $N^{\circ}$ 11: ¿Qué opinión tienes de los materiales didácticos demostrativos?

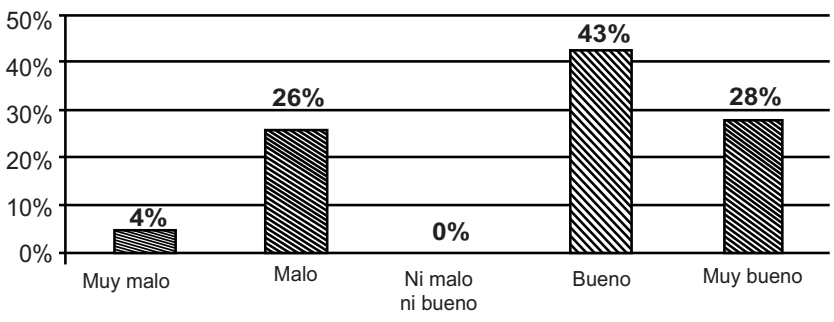

En laFigura 11 se muestra que el $4 \%$ cree que los materiales didácticos demostrativos son muy malos, un $26 \%$ son malos, un $43 \%$ son buenos y un $28 \%$ los materiales demostrativos son muy buenos. La mayoría de estudiantes opinan que es buena la enseñanza de las propiedades matemáticas con los materiales didácticos demostrativos.

Con los resultados de la encuesta se concluyó que los estudiantes de la Universidad Santiago Antúnez de Mayolo poco sabían de las demostraciones matemáticas y de los materiales didácticos concretos usados para demostrar propiedades matemáticas.

Análisis del pretest y postest tanto del grupo control y experimental. aprendizaje de la matemática en los estudiantes de la Escuela Profesional de Educación Primaria de la FCSEC UNASAM-2017,

Esta conclusión queda demostrada, en función a la comparación de medias en los momentos dados (pretest y 
postest), entre los grupos control y experimental.

Tabla $\mathbf{N}^{\circ}$ 2. Manipulacion de materiales concretos $y$ capacidad de matemáticas

\begin{tabular}{lccc|} 
& Pruebas de chi-cuadrado & \\
& Valor & gl & $\begin{array}{c}\text { Significación asintótica } \\
\text { (bilateral) }\end{array}$ \\
\hline $\begin{array}{l}\text { Chi-cuadrado de } \\
\text { Pearson }\end{array}$ & 22,143 & 3 &, 023 \\
\hline $\begin{array}{l}\text { Razón de } \\
\text { verosimilitud }\end{array}$ & 29,252 & 3 & \\
\hline $\begin{array}{l}N \text { de casos } \\
\text { válidos }\end{array}$ & 46 & & \\
\hline
\end{tabular}

Según la tabla 2 podemos establecer que si existe dependencia entre las variables manipulación de materiales concretas y la capacidad de matematizar situaciones en los alumnos de la escuela profesional de Educación primaria de la FCSEC UNASAM - 2017.

Esta conclusión queda evidenciada mediante la prueba de Chi cuadrado, cuyo $p$-valor obtenido es menor al grado de significancia $(0.023<0.05)$, por la cual se acepta la hipótesis alternativa, resaltando que existe una correlación estadísticamente significativa entre las variables.

Tabla $\mathbf{N}^{\circ}$ 3. Materiales concretos demostrativo y capacidad de razonar

\begin{tabular}{|c|c|c|c|}
\hline \multicolumn{4}{|c|}{ Pruebas de chi-cuadrado } \\
\hline & Valor & gl & $\begin{array}{c}\text { Significación asintótica } \\
\text { (bilateral) }\end{array}$ \\
\hline $\begin{array}{l}\text { Chi-cuadrado de } \\
\text { Pearson }\end{array}$ & 26,056 & 2 & 001 \\
\hline $\begin{array}{l}\text { Razón de } \\
\text { verosimilitud }\end{array}$ & 34,540 & 2 &, 000 \\
\hline $\begin{array}{l}\mathrm{N} \text { de casos } \\
\text { válidos }\end{array}$ & 46 & & \\
\hline
\end{tabular}

Por medio la tabla 3 podemos establecer que si existe dependencia entre las variables materiales concretos demostrativos y la capacidad de razonar y argumentar, generando ideas matemáticas en los alumnos de la escuela profesional de Educación primaria de la FCSEC UNASAM - 2017.

Esta conclusión queda evidenciada mediante la prueba de Chi cuadrado, cuyo $p$-valor obtenido es menor al grado de significancia $(0.001<0.05)$, por la cual se acepta la hipótesis alternativa, resaltando que existe una correlación estadísticamente significativa entre las variables.

Tabla $\mathbf{N}^{\circ} 4$. Demostracion atravez de materiales concreto

\begin{tabular}{|c|c|c|c|}
\hline \multicolumn{4}{|c|}{ Pruebas de chi-cuadrado } \\
\hline & Valor & $\mathrm{gl}$ & $\begin{array}{c}\text { Significación asintótica } \\
\text { (bilateral) }\end{array}$ \\
\hline $\begin{array}{l}\text { Chi-cuadrado de } \\
\text { Pearson }\end{array}$ & 26,610 & 3 &, 005 \\
\hline $\begin{array}{l}\text { Razón de } \\
\text { verosimilitud }\end{array}$ & 35,110 & 3 &, 000 \\
\hline $\begin{array}{l}\mathrm{N} \text { de casos } \\
\text { válidos }\end{array}$ & 46 & & \\
\hline
\end{tabular}

En la tabla 4 podemos establecer que, si existe dependencia entre las variables demostración a través de los materiales concretos, por ser más accesible, sencilla, motivadora y apropiada que la demostración formal y el desarrollo de la capacidad de comunicar y representar ideas matemáticas de los alumnos de la Escuela Profesional de Educación Primaria de la FCSEC UNASAM - 2017.

Esta conclusión queda evidenciada mediante la prueba de Chi cuadrado, cuyo $p$-valor obtenido es menor al grado de significancia $(0.005<0.05)$, por la cual se acepta la hipótesis alternativa, resaltando que existe una correlación estadísticamente significativa entre las variables.

\section{DISCUSIÓN DE LOS RESULTADOS}

Los resultados evidenciaron que si hay una influencia positiva de los materiales didácticos demostrativos hacia la mejora de los aprendizajes de la matemática.

Los estudiantes de grupo experimental en el pos test se observaron que reforzaron los contenidos matemáticos que se trabajaron, puesto que pudieron experimentar las propiedades abstractas de una forma más natural, lógica y divertida, ya que las propiedades tuvieron más sentido y significancia al manipular, ellos mismos las propiedades de los triángulos y al demostrarlos visualmente. Por consiguiente, el aprendizaje fue más significativo, y se logró aprendizajes duraderos. La argumentación que dieron de las propiedades de los triángulos, de los cuadriláteros y de los polígonos fue más coherente, las recordaron más claramente y seleccionaron mejor al aplicarlos en la resolución de problemas.

Además podría mencionar que los resultados obtenidos en la T-student (Tabla 1), para $\infty=0,05$ se obtuvo el $p$-valor de 0.27 y 0.19 para el pretest y $0.30,0.07$ para el postest adoptando la decisión de aceptar la hipótesis alterna, por lo cual se puede afirmar que: si se utiliza adecuadamente los materiales didácticos demostrativos en las sesiones de clase mejorará el aprendizaje de la matemática en Los estudiantes de la Escuela Profesional de Educación Primaria de la FCSEC UNASAM - 2017; estos resultados son suficientemente satisfactorios por cuanto al logro de los objetivos de investigación.

En las pruebas chi-cuadrado que se realizaron para medir la dependencia de las variables se obtuvieron los siguientes resultados:

La prueba de Chi cuadrado (Tabla 2), se evidencia mediante $p$-valor obtenido es menor al grado de significancia $(0.023<$ 0.05 ), por la cual se afirma que existe una dependencia entre las variables manipulación de materiales concretas y la capacidad de matematizar situaciones en los estudiantes universitarios de la Facultad de Educación.

La prueba de Chi cuadrado (Tabla 3), se evidencia mediante $p$-valor obtenido es menor al grado de significancia $(0.001<$ $0.05)$, por la cual se afirma que existe una dependencia entre las variables materiales concretos demostrativos y la capacidad de razonar y argumentar, generando ideas matemáticas en los estudiantes universitarios de la Facultad de Educación.

La prueba de Chi cuadrado (Tabla 3), se evidencia mediante $p$-valor obtenido es menor al grado de significancia $(0.005<$ 0.05 ), por la cual se afirma que existe una dependencia entre las variables demostración a través de los materiales concretos, por ser más accesible, sencilla, motivadora y apropiada que la demostración formal y el desarrollo de la capacidad de comunicar y representar ideas matemáticas en los estudiantes universitarios de la Facultad de Educación. 


\section{CONCLUSIONES}

1)Los efectos que tiene los materiales didácticos demostrativos son la obtención de mejores aprendizajes en las matemáticas, así como en las capacidades de argumentación, retención de los conocimientos, interpretación y en la aplicación de las propiedades en la resolución de problemas como se demuestra en la prueba de la hipótesis general.

2)La interacción de los materiales didácticos demostrativos hechos con tecnoport se mejoró la capacidad de matematizar: identificando, interpretando y evaluando situaciones problemáticas por parte de los estudiantes de la Escuela Profesional de Educación Primaria de la Universidad "Santiago Antúnez de Mayolo"

3)La manipulación de los bloques de tecnopor que representan a las figuras geométricas, influye favorablemente en la capacidad de matematizar situaciones, usando las propiedades geométricas y en la aplicación en los problemas, como se demuestra en la prueba de la primera hipótesis específica.

4)La elaboración de materiales didácticos demostrativos influye favorablemente en la capacidad de razonar y argumentar generando ideas matemáticas en los estudiantes, como se demuestra en la prueba de la segunda hipótesis específica.
5)La demostración a través de los bloques de tecnopor desarrollan la capacidad de comunicar y representar ideas matemáticas en los estudiantes, como se demuestra en la prueba de la tercera hipótesis específica.

\section{AGRADECIMIENTOS}

A todas las personas que hicieron posible la realización de este proyecto de investigación, a los directivos de la universidad Nacional "Santiago Antúnez de Mayolo" por su buena disposición en la aplicación del proyecto y a mis familiares que siempre estuvieron allí dándome las fuerzas y el aliento para seguir.

\section{REFERENCIAS}

Balachef N.(1998). Contract and custom: two regisers of didactical interractions. the matematics educator. 9(2)

Hanna, G. y Janhke, H. (1996). "Proof and proving" Estados Unidos: Editorial Proof

Valerio, F. (2000), "Nuevas Estrategias Didácticas Activas en las Ciencias Sociales". Perú: Editorial Killa.

Van Hiele, P. (1986) "Structure and insight”. New York: Editorial Academic Press. 